\title{
Asymptotic Expansions for the Discretization Error of Least Squares Solutions of Linear Boundary Value Problems
}

\author{
By Klaus Böhmer and John Locker
}

\begin{abstract}
For determining least squares solutions of linear boundary value problems, the method of regularization provides uniquely solvable boundary value problems, which are solved with difference methods. The determination of the coefficients in an asymptotic expansion of the discretization error in powers of the regularization and discretization parameters $\alpha$ and $h$, respectively, is an ill-posed problem. We present here an asymptotic expansion of this type and discuss the numerical implications for Richardson extrapolation, thereby establishing for the first time methods of arbitrarily high order.
\end{abstract}

1. Introduction. For the numerical solution of well-posed boundary value problems via difference approximations, the existence of an asymptotic expansion of the discretization error in powers of the stepsize $h$ is a most important fact. Expansions of this type are basic for Richardson extrapolation (see [19]), for deferred corrections (see Pereyra [16], [17], Keller and Pereyra [10], Russell [20], and Skeel [21]), and for discrete Newton methods (see Böhmer [2], [3]). For ill-posed problems the situation is more complex.

In this paper we compute the least squares minimal-norm solution of an ill-posed linear boundary value problem by combining regularization and difference methods. For the method of regularization the ill-posed problem is transformed into a family of "neighboring" well-posed problems involving a regularization parameter $\alpha$, and then the limit is taken as $\alpha$ tends to 0 . This approach was historically introduced by Phillips [18] and Tikhonov [23], [24] to overcome the numerical difficulties in solving integral equations of the first kind. In [12], [13], [14] Locker and Prenter applied this method, combined with finite element approximations, to first-kind integral equations and differential equations. With a stepsize parameter $h$ for the finite element method of order $k$, they obtain $O\left(h^{k} / \alpha^{2}\right)$ for the error. Natterer [15] used projection methods on the original ill-posed equation, employing an appropriate fixed $h=h_{\mathrm{opt}}$, which is naturally available from the projection method as regularization parameter. A limiting $h_{\text {opt }}$ tending to zero and an asymptotic expansion do not make sense in this context. In combining regularization and difference methods we obtain an asymptotic expansion for the "regularized" discretization error in powers of the regularization parameter $\alpha$ and the stepsize $h$.

Received June 5, 1984; revised July 15, 1987.

1980 Mathematics Subject Classification (1985 Revision). Primary 34B05, 34E05, $65 \mathrm{~L} 60$.

Key words and phrases. Least squares solutions, regularization, ill-posed problems, asymptotic expansions for discretization, Richardson extrapolation. 
Throughout this paper we work in the real Hilbert space $L^{2}[0,1]$ with its standard inner product $(\cdot, \cdot)$ and norm $\|\cdot\|$. Let

$$
L_{0}=\sum_{\nu=0}^{n} A_{\nu} D^{\nu}
$$

be an $n$ th-order formal differential operator on the interval $[0,1]$, let

$$
B_{i} x=\sum_{\nu=0}^{n-1}\left[b_{i \nu}(0) D^{\nu} x(0)+b_{i \nu}(1) D^{\nu} x(1)\right], \quad i=1, \ldots, k,
$$

be a set of $k(0 \leq k \leq 2 n)$ linearly independent boundary operators, and let $L$ be the $n$ th-order differential operator in $L^{2}[0,1]$ defined by

$$
L:\left\{\begin{array}{l}
\mathscr{D}(L):=\left\{x \in H^{n}[0,1] \mid B_{i} x=0, i=1, \ldots, k\right\} \rightarrow L^{2}[0,1] \\
x \mapsto L x:=L_{0} x,
\end{array}\right.
$$

where $H^{n}[0,1]$ is the Sobolev space consisting of all $x \in C^{n-1}[0,1]$ with $x^{(n-1)}$ absolutely continuous on $[0,1]$ and $x^{(n)} \in L^{2}[0,1]$. For a given $y \in L^{2}[0,1]$ we determine the least squares minimal-norm solution of the linear boundary value problem

$$
L x=y
$$

by the method of regularization, using the identity operator $I$ as regularization operator.

For each $\alpha \in \mathbf{R}, \alpha \neq 0$, let $G_{\alpha}$ be the functional defined on $\mathscr{D}(L)$ by $G_{\alpha} x:=$ $\|L x-y\|^{2}+\alpha^{2}\|x\|^{2}$. In regularization one shows that there exists a unique solution $x_{\alpha} \in \mathscr{D}(L)$ to the minimization problem

$$
G_{\alpha} x_{\alpha}=\inf _{x \in \mathscr{D}(L)} G_{\alpha} x
$$

and that as $\alpha \rightarrow 0$ the $x_{\alpha}$ converge to the least squares solution $x_{0} \in \mathscr{D}(L)$ of $(1.1)$ having minimal norm $\|\cdot\|$. The adjoint operator $L^{*}$ is also an $n$ th-order differential operator in $L^{2}[0,1]$ determined by the formal adjoint $L_{0}^{*}$ and by adjoint boundary operators $B_{i}^{*}, i=k+1, \ldots, 2 n$. In terms of $L$ and $L^{*}$, for each $\alpha \neq 0$ the $x_{\alpha}$ in (1.2) is characterized by (see [14])

$$
\left\{\begin{array}{l}
x_{\alpha} \in \mathscr{D}(L), L x_{\alpha}-y \in \mathscr{D}\left(L^{*}\right), \text { and } \\
L^{*}\left(L x_{\alpha}-y\right)+\alpha^{2} x_{\alpha}=0 .
\end{array}\right.
$$

Other equivalent characterizations are possible (see [5]).

In Section 2 we establish the power series expansion

$$
x_{\alpha}=\sum_{\mu=0}^{\infty} \alpha^{2 \mu} e_{2 \mu}, \quad e_{0}=x_{0}
$$

which converges with respect to the $H^{2 n}$-Sobolev norm for $\alpha$ sufficiently small. Here the $e_{2 \mu}$ are independent of $\alpha$. The expansion (1.4) is based on the series representation

$$
\left(L^{*} L+\alpha^{2} I\right)^{-1}=\frac{1}{\alpha^{2}} P+\sum_{i=0}^{\infty}(-1)^{i} \alpha^{2 i} K^{i+1}
$$


where $P$ is the orthogonal projection onto the null space of $L$ and $K$ is the generalized inverse of $L^{*} L$. In that section it is also shown that all these series in powers of $\alpha^{2}$ represent asymptotic expansions.

In Section 3 we use compact symmetric difference schemes (see, e.g., Keller and Pereyra [10]) to solve (1.3). Stability and convergence results for the difference approximate $x_{\alpha}^{h}$ are derived there. For sufficiently small $\alpha$ and $h<h_{\alpha}$ we even obtain in Section 4 an asymptotic expansion of the discretization error of the form

$$
x_{\alpha}^{h}(t)=\sum_{\nu=0}^{q-1} h^{2 \nu} \sum_{\mu=0}^{j_{\nu}} \alpha^{2(\mu-\nu)} f_{2 \mu, 2 \nu}(t)+O\left(\frac{h^{2 q}}{\alpha^{2 q}}\right)
$$

for grid points $t$ and sufficiently large $j_{\nu}$. The coefficients $f_{2 \mu, 2 \nu}$ are independent of $\alpha$ and $h$, and $f_{2 \mu, 0}=e_{2 \mu}$ as in (1.4). See Eqs. (4.13) and (5.1) for details. Unless very specific information for $(1.1)$ is provided, namely $\mathscr{N}(L)=\{0\}$, for a combination of regularization and difference methods the $\alpha^{-2 \nu}$ terms in (1.6) are unavoidable.

The development of these results for $\mathscr{N}(L) \neq\{0\}$ is strongly aggravated by the fact that (1.3) represents a whole family of boundary value problems, with the norms of the associated operators $\left(L^{*} L+\alpha^{2} I\right)^{-1}$ exploding at the rate $C / \alpha^{2}$ as $\alpha \rightarrow 0$. Although the determination of a minimal-norm least squares solution to (1.1) is a well-posed problem, numerical methods do not inherit this property. This becomes apparent in the $O\left(h^{k} / \alpha^{2}\right)$ result of Locker and Prenter and the need for choosing a specific $h_{\text {opt }}$ in Natterer. Furthermore, the computation of an asymptotic expansion for the discretization error is an ill-posed problem because of (1.5), and to our knowledge, it has not been done before. To this end, the operator $\left(L^{*} L+\alpha^{2} I\right)^{-1}$ has to be applied $q-1$ times, a process that finally yields (1.6).

There are many benefits to our approach. Whereas the usual methods are limited to fixed-order discretization methods, where $\alpha$ and $h$ have to be fitted, we may directly use (1.6) to define a class of discretization methods of variable order, e.g., via Richardson extrapolation or discrete Newton methods or some other type of defect corrections. Numerical examples, presented in Section 5, show very clearly these nice features. For the most interesting, and in our context, the usual case where $\mathscr{N}(L) \neq\{0\}$, we restrict the numerical experiments to the case $\alpha=\sqrt{\gamma h}$ with a fixed constant $\gamma$, obtaining from (1.6) an asymptotic expansion in powers of $h$ (instead of powers of $h^{2}$ and $\alpha^{2}$ ) of the form

$$
x^{h}(t):=x_{\sqrt[\gamma]{\gamma h}}^{h}(t)=x_{0}(t)+h e_{1}^{*}(t)+h^{2} e_{2}^{*}(t)+\cdots .
$$

As always, high-order methods are worthwhile only in smooth situations where high accuracy is required. In such a situation these variable-order methods based on asymptotic expansions are excellent tools to obtain high accuracy in comparatively little computation time.

In this paper we omit some of the proofs and many technicalities, and we refer the interested reader to [5]. 
2. Power Series and Asymptotic Expansions. Assume that the coefficients $A_{\nu}$ of $L_{0}$ are sufficiently smooth to form $L_{0}^{*} L_{0}$ and

$$
B_{i} x:=B_{i}^{*} L_{0} x=\sum_{\nu=0}^{2 n-1}\left[b_{i \nu}(0) D^{\nu} x(0)+b_{i \nu}(1) D^{\nu} x(1)\right], \quad i=k+1, \ldots, 2 n .
$$

In solving (1.1) and (1.3) we will use the differential operator $L^{*} L$ given by

$$
L^{*} L:\left\{\begin{array}{l}
\mathscr{D}\left(L^{*} L\right)=\left\{x \in H^{2 n}[0,1] \mid B_{i} x=0, i=1, \ldots, 2 n\right\} \rightarrow L^{2}[0,1] \\
x \mapsto L^{*} L x=L_{0}^{*} L_{0} x,
\end{array}\right.
$$

together with the generalized inverses of $L$ and $L^{*} L$ :

$$
L^{\dagger}:\left\{\begin{array}{l}
\mathscr{D}\left(L^{\dagger}\right):=L^{2}[0,1] \rightarrow \mathscr{D}(L) \\
x \mapsto L^{\dagger} x:=\left[L \mid \mathscr{D}(L) \cap \mathscr{N}(L)^{\perp}\right]^{-1}(I-Q) x
\end{array}\right.
$$

and

$$
K:=\left(L^{*} L\right)^{\dagger}:\left\{\begin{array}{l}
\mathscr{D}(K):=L^{2}[0,1] \rightarrow \mathscr{D}\left(L^{*} L\right) \\
x \mapsto K x:=\left[L^{*} L \mid \mathscr{D}\left(L^{*} L\right) \cap \mathscr{N}\left(L^{*} L\right)^{\perp}\right]^{-1}(I-P) x
\end{array}\right.
$$

where $P, I-P, Q$, and $I-Q$ are the orthogonal projections onto the subspaces $\mathscr{N}(L)=\mathscr{N}\left(L^{*} L\right), \mathscr{R}\left(L^{*}\right)=\mathscr{R}\left(L^{*} L\right), \mathscr{N}\left(L^{*}\right)$, and $\mathscr{R}(L)$, respectively. Let

$$
x_{0}:=L^{\dagger} y \in \mathscr{D}(L) \cap \mathscr{N}(L)^{\perp},
$$

the least squares minimal-norm solution of (1.1). Utilizing these operators, we are able to construct a power series expansion of the regularization function $x_{\alpha}$ in powers of $\alpha^{2}$ and in terms of the least squares solution $x_{0}$.

For $\mathscr{N}(L)=\{0\}$ or $P=0$, we have $K=\left(L^{*} L\right)^{-1}$. In this case we might want to avoid regularization completely, and instead of $L x=y$ study the uniquely solvable problem

$$
L^{*}(L x-y)=0 .
$$

Assume that $y \in H^{n}[0,1]$, which implies that $x_{0}, x_{\alpha} \in H^{2 n}[0,1]$. Then (1.3) can be rewritten as

$$
\left\{\begin{array}{l}
L_{0}^{*} L_{0} x_{\alpha}+\alpha^{2} x_{\alpha}=L_{0}^{*} y \\
B_{i} x_{\alpha}=0, \quad i=1, \ldots, k \\
B_{i} x_{\alpha}=B_{i}^{*} y, \quad i=k+1, \ldots, 2 n .
\end{array}\right.
$$

In the next two sections we will numerically solve (2.1) by using finite difference methods. This will require replacing $(2.1)$ by

$$
\left\{\begin{array}{l}
L_{0}^{*} L_{0} w_{\alpha}+\alpha^{2} w_{\alpha}=z \\
B w_{\alpha}:=\left(B_{i} w_{\alpha}\right)_{i=1}^{2 n}=\beta:=\left(\beta_{i}\right)_{i=1}^{2 n},
\end{array}\right.
$$

where $z \in L^{2}[0,1]$ and $\beta_{1}, \ldots, \beta_{2 n}$ are constants. We now proceed to solve (2.2), expanding $w_{\alpha}$ in a power series.

Let $b_{i \nu}(0):=b_{i \nu}(1):=0$ for $i=1, \ldots, k$ and $\nu=n, \ldots, 2 n-1$; let $N$ be the $2 n \times 4 n$ boundary matrix

$$
N:=\left(b_{i \nu}(0), b_{i \nu}(1), \nu=0,1, \ldots, 2 n-1\right)_{i=1}^{2 n},
$$


which has rank $2 n$; and let $N^{\dagger}$ be the Moore-Penrose generalized inverse of $N$. Next, let $H$ be the Hermite interpolation operator defined by

$$
H:\left\{\begin{array}{l}
\mathbf{R}^{4 n} \rightarrow \pi_{4 n-1}=\{\text { polynomials of degree } \leq 4 n-1\}, \\
d:=\left(d_{\nu 0}, d_{\nu 1}, \nu=0,1, \ldots, 2 n-1\right)^{T} \mapsto H d:=p \text { such that } p^{(\nu)}(0)=d_{\nu 0}, \\
p^{(\nu)}(1)=d_{\nu 1}, \quad \nu=0,1, \ldots, 2 n-1 .
\end{array}\right.
$$

Finally, let

$$
p:=H N^{\dagger} \beta, \quad v_{\alpha}:=w_{\alpha}-p, \quad z_{0}:=z-L_{0}^{*} L_{0} p, \quad z_{\alpha}:=z_{0}-\alpha^{2} p .
$$

The polynomial $p$ depends continuously on $\beta$ and satisfies $B p=\beta$, and $v_{\alpha} \in$ $\mathscr{D}\left(L^{*} L\right)$ with

$$
\left(L^{*} L+\alpha^{2} I\right) v_{\alpha}=z-L_{0}^{*} L_{0} p-\alpha^{2} p=z_{0}-\alpha^{2} p=z_{\alpha} .
$$

Therefore, $v_{\alpha}=\left(L^{*} L+\alpha^{2} I\right)^{-1} z_{\alpha}$ and

$$
w_{\alpha}=\left(L^{*} L+\alpha^{2} I\right)^{-1} z_{\alpha}+p=\left(L^{*} L+\alpha^{2} I\right)^{-1}\left(z_{0}-\alpha^{2} p\right)+p
$$

and consequently, we need a power series expansion for the operator $\left(L^{*} L+\alpha^{2} I\right)^{-1}$.

With this goal in mind, let $\mathscr{B}\left(L^{2}[0,1]\right)$ denote the Banach space of all bounded linear operators on $L^{2}[0,1]$ with norm

$$
\|T\|_{L^{2}}=\sup _{\|x\|=1}\|T x\| .
$$

For the Sobolev space $H^{n}[0,1]$ we introduce the $H^{n}$-Sobolev norm

$$
\|x\|_{H^{n}}:=\sum_{i=0}^{n-1}\left\|x^{(i)}\right\|_{\infty}+\left\|x^{(n)}\right\| .
$$

It is well known that $\mathscr{D}\left(L^{*} L\right)$ becomes a Banach space under the $H^{2 n}$-Sobolev norm $\|\cdot\|_{H^{2 n}}$. Let $\mathscr{X}$ denote the Banach space of all bounded linear operators from $L^{2}[0,1]$ under the $L^{2}$-structure into $\mathscr{D}\left(L^{*} L\right)$ under the $H^{2 n}$-Sobolev structure, with norm

$$
\|T\|_{H^{0,2 n}}=\sup _{\|x\|=1}\|T x\|_{H^{2 n}} .
$$

Clearly, the linear operators $\left(L^{*} L+\alpha^{2} I\right)^{-1}, P$, and $K^{i+1}, i=0,1,2, \ldots$, belong to both $\mathscr{B}\left(L^{2}[0,1]\right)$ and $\mathscr{X}$.

For the generalized inverse $K=\left(L^{*} L\right)^{\dagger}=L^{\dagger}\left(L^{*}\right)^{\dagger}$, considered as an operator in $\mathscr{B}\left(L^{2}[0,1]\right)$, we know that $I+\alpha^{2} K$ is invertible for $|\alpha|<\|K\|_{L^{2}}^{-1 / 2}$, and $K P=0$ and $\left(I+\alpha^{2} K\right) P=P$, and hence,

$$
\left(I+\alpha^{2} K\right)^{-1} P=P .
$$

Applying the operators $P$ and $K$ to the equation

$$
\left(L^{*} L+\alpha^{2} I\right)\left(L^{*} L+\alpha^{2} I\right)^{-1}=I,
$$

we obtain

$$
P\left(L^{*} L+\alpha^{2} I\right)^{-1}=\frac{1}{\alpha^{2}} P
$$

and

$$
\left(I-P+\alpha^{2} K\right)\left(L^{*} L+\alpha^{2} I\right)^{-1}=K .
$$


In view of (2.6) we can rewrite $(2.7)$ as

$$
\left(I+\alpha^{2} K\right)\left(L^{*} L+\alpha^{2} I\right)^{-1}=\frac{1}{\alpha^{2}} P+K,
$$

and finally, by (2.5) this becomes

$$
\left(L^{*} L+\alpha^{2} I\right)^{-1}=\frac{1}{\alpha^{2}} P+K\left(I+\alpha^{2} K\right)^{-1}
$$

for $0<|\alpha|<\|K\|_{L^{2}}^{-1 / 2}$. Here and in the sequel the negative powers of $\alpha^{2}$ will occur if and only if $P \neq 0$, or equivalently, $\mathscr{N}(L) \neq\{0\}$, the exception being when $P$ is applied to special elements belonging to $\mathscr{N}(P)=\mathscr{N}(L)^{\perp}=\mathscr{R}\left(L^{*}\right)$, e.g., as in (2.18).

Next, we expand $\left(I+\alpha^{2} K\right)^{-1}$ in a Neumann type expansion in $\mathscr{B}\left(L^{2}[0,1]\right)$. Define

$$
\alpha_{0}:=\left(2\|K\|_{L^{2}}\right)^{-1 / 2},
$$

and let us assume throughout the rest of the paper that $0<|\alpha|<\alpha_{0}$. Clearly, $\alpha^{2}\|K\|_{L^{2}} \leq \alpha^{2} / \alpha_{0}^{2}$ and $1 /\left(1-\alpha^{2}\|K\|_{L^{2}}\right) \leq 2$, and

$$
\left(I+\alpha^{2} K\right)^{-1}=\sum_{i=0}^{\infty}(-1)^{i} \alpha^{2 i} K^{i} \quad \text { in } \mathscr{B}\left(L^{2}[0,1]\right),
$$

with the error estimate

$$
\left\|\left(I+\alpha^{2} K\right)^{-1}-\sum_{i=0}^{j}(-1)^{i} \alpha^{2 i} K^{i}\right\|_{L^{2}} \leq 2\left(\alpha^{2} / \alpha_{0}^{2}\right)^{j+1}
$$

for $j=0,1,2, \ldots$ To simplify the notation in the sequel, we set

$$
K_{-2}:=P \quad \text { and } \quad K_{2 i}:=(-1)^{i} K^{i+1}, \quad i=0,1,2, \ldots
$$

Then substituting (2.9) into (2.8), we obtain the expansion

$$
\left(L^{*} L+\alpha^{2} I\right)^{-1}=\sum_{i=-1}^{\infty} \alpha^{2 i} K_{2 i} \quad \text { in } \mathscr{X},
$$

with the error estimate

$$
\left\|\left(L^{*} L+\alpha^{2} I\right)^{-1}-\sum_{i=-1}^{j} \alpha^{2 i} K_{2 i}\right\|_{H^{0,2 n}} \leq 2\|K\|_{H^{0,2 n}}\left(\alpha^{2} / \alpha_{0}^{2}\right)^{j+1}
$$

for $j=0,1,2, \ldots$ Note that $(2.12)$ is also valid for $j=-1$ by (2.8) and (2.9). In addition, (2.11) yields the bound

$$
\left\|\left(L^{*} L+\alpha^{2} I\right)^{-1}\right\|_{H^{0,2 n}} \leq \frac{1}{\alpha^{2}}\|P\|_{H^{0,2 n}}+2\|K\|_{H^{0,2 n}} .
$$

The final step is to combine (2.4) and (2.11). Indeed, if we set

$$
\left\{\begin{array}{l}
e_{-2}:=K_{-2} z_{0}=P z_{0} \\
e_{0}:=K_{0} z_{0}+\left(I-K_{-2}\right) p=K z_{0}+(I-P) p \\
e_{2 i}:=K_{2 i} z_{0}-K_{2(i-1)} p=(-1)^{i} K^{i+1} z_{0}-(-1)^{i-1} K^{i} p, \quad i=1,2, \ldots
\end{array}\right.
$$


then we get the expansion

$$
w_{\alpha}=\sum_{i=-1}^{\infty} \alpha^{2 i} e_{2 i} \quad \text { in } H^{2 n}[0,1],
$$

with the error estimate (use (2.12))

$$
\left\|w_{\alpha}-\sum_{i=-1}^{j} \alpha^{2 i} e_{2 i}\right\|_{H^{2 n}} \leq C\left(\alpha^{2} / \alpha_{0}^{2}\right)^{j+1}\left(\|z\|+\|\beta\|_{0}\right)
$$

for $j=0,1,2, \ldots$ In this inequality and in the sequel, $C$ denotes a generic constant which is independent of the parameters $\alpha$ and $h$, and $\|\cdot\|_{0}$ denotes a fixed norm on $\mathbf{R}^{2 n}$.

Remark 2.1. The $w_{\alpha}$ need not converge as $\alpha \rightarrow 0$. For example, if $z \in \mathscr{N}(L)$ with $z \neq 0$ and $\beta=0$, then $p=0, P z_{0}=z$,

$$
w_{\alpha}=\frac{1}{\alpha^{2}} z, \quad \text { and } \quad\left\|w_{\alpha}\right\|_{H^{2 n}} \rightarrow \infty \quad \text { as } \alpha \rightarrow 0 .
$$

This type of behavior has already been observed in [9].

Special Case. (2.2) reduces to (2.1): $w_{\alpha}=x_{\alpha}$. In this special case we have

$$
z=L_{0}^{*} y ; \beta_{i}=0, i=1, \ldots, k ; \beta_{i}=B_{i}^{*} y, i=k+1, \ldots, 2 n .
$$

Consequently, $B_{i}^{*}\left(y-L_{0} p\right)=\beta_{i}-B_{i} p=0$ for $i=k+1, \ldots, 2 n$, so $y-L_{0} p \in \mathscr{D}\left(L^{*}\right)$, $z_{0}=L_{0}^{*} y-L_{0}^{*} L_{0} p \in \mathscr{R}\left(L^{*}\right)$, and

$$
e_{-2}=P z_{0}=0 \text {. }
$$

Also, $B_{i} p=\beta_{i}=0$ for $i=1, \ldots, k$, so $p \in \mathscr{D}(L)$. Since

$$
K L^{*}=L^{\dagger}\left(L^{*}\right)^{\dagger} L^{*}=L^{\dagger}(I-Q)=L^{\dagger} \quad \text { on } \mathscr{D}\left(L^{*}\right),
$$

it follows that

$$
e_{0}=(I-P) p+K L^{*}(y-L p)=(I-P) p+L^{\dagger} y-L^{\dagger} L p=L^{\dagger} y
$$

or

$$
e_{0}=L^{\dagger} y=x_{0}
$$

We conclude that the regularization function $x_{\alpha}$ has the power series expansion (1.4) with the error estimate

$$
\left\|x_{\alpha}-\sum_{i=0}^{j} \alpha^{2 i} e_{2 i}\right\|_{H^{2 n}} \leq C\left(\alpha^{2} / \alpha_{0}^{2}\right)^{j+1}\|y\|_{H^{n}}
$$

for $j=0,1,2, \ldots$ In particular, for $j=0$ we get the well-known estimate (see [14])

$$
\left\|x_{\alpha}-x_{0}\right\|_{H^{2 n}} \leq C \alpha^{2}\|y\|_{H^{n}} .
$$

Since the right-hand sides of (2.12), (2.16), and (2.20) are $O\left(\alpha^{2 j+2}\right)$ for fixed $j$, the corresponding power series represent asymptotic expansions. A similar statement is true for the series in Sections 3 and 4. 
Remark 2.2. In our numerical work it will be necessary to use smoother classes of functions than $L^{2}[0,1]$ and $H^{2 n}[0,1]$. Indeed, for $0 \leq l<\infty$ consider the Banach space $C^{l}[0,1]$ with norm

$$
\|x\|_{C^{l}}:=\sum_{i=0}^{l}\left\|x^{(i)}\right\|_{\infty} .
$$

Clearly $\mathscr{D}\left(L^{*} L\right) \cap C^{2 n+l}[0,1]$ is a Banach space under the norm $\|\cdot\|_{C^{2 n+l}}$. Let $\mathscr{B}\left(C^{l}[0,1]\right)$ and $\mathscr{Z}_{l}$ be the respective counterparts of $\mathscr{B}\left(L^{2}[0,1]\right)$ and $\mathscr{X}$ with norms

$$
\|T\|_{C^{l}}=\sup _{\|x\|_{C^{l}}=1}\|T x\|_{C^{l}}
$$

and

$$
\|T\|_{C^{l, 2 n+l}}=\sup _{\|x\|_{C^{l}=1}}\|T x\|_{C^{2 n+l}} .
$$

The linear operators in $(2.11)$ all belong to $\mathscr{Z}_{l}$ when suitably restricted. Thus, by similar arguments, the above results for $\left(L^{*} L+\alpha^{2} I\right)^{-1}$ are valid in $\mathscr{Z}_{l}$ with the norm $\|\cdot\|_{C^{l, 2 n+l}}$ replacing $\|\cdot\|_{H^{0,2 n}}$, and assuming $z \in C^{l}[0,1]$ and $y \in C^{n+l}[0,1]$, the results for the $w_{\alpha}$ and the $x_{\alpha}$ are valid in $C^{2 n+l}[0,1]$ with the norm $\|\cdot\|_{C^{2 n+l}}$ replacing $\|\cdot\|_{H^{2 n}}$ (see [5]).

3. Stability and Convergence for Finite Difference Methods. Based on (1.3) and (2.1), our aim is to compute finite difference approximations $x_{\alpha}^{h}$ for $x_{\alpha}$. Let

$$
F_{\alpha} x:=\left\{\begin{array}{l}
M_{\alpha} x:=L_{0}^{*} L_{0} x+\alpha^{2} x:=\sum_{\nu=0}^{2 n}\left(a_{\nu}+\alpha^{2} \delta_{\nu 0}\right) D^{\nu} x \\
B x:=\left(B_{i} x=\sum_{\nu=0}^{2 n-1}\left[b_{i \nu}(0) D^{\nu} x(0)+b_{i \nu}(1) D^{\nu} x(1)\right]\right)_{i=1}^{2 n} .
\end{array}\right.
$$

Then Eq. (2.1) becomes

$$
F_{\alpha} x_{\alpha}=\left(\begin{array}{c}
L_{0}^{*} y \\
\beta_{y}
\end{array}\right) \quad \text { with } \beta_{y}:=\left(\begin{array}{l}
\beta_{i}=0, i=1, \ldots, k \\
\beta_{i}=B_{i}^{*} y, i=k+1, \ldots, 2 n
\end{array}\right) .
$$

To discretize (3.2), we introduce a stepsize $h$, an equidistant grid $\mathbf{G}^{h}$, and $\mathbf{G}_{0}^{h}:=$ $\mathbf{G}^{h} \cap[0,1]$ by

$$
h:=\frac{1}{m}, \quad \mathbf{G}^{h}:=\left\{t_{i}:=i h, i=-n,-n+1, \ldots, 0,1, \ldots, m, \ldots, m+n\right\} .
$$

To simplify matters and to allow Richardson extrapolation, we have changed the $\mathrm{G}^{h}$ and the $B_{i}^{h}$ given below from those used in [5] and [10]. As a consequence of $\mathbf{G}^{h} \not \subset[0,1]$, we have to use extensions $\bar{z}$ for functions $z$ to an appropriate larger interval $[-\delta, 1+\delta]$. The details for the extension procedures are given in [5], where it is shown that the extension operators are continuous; we do not distinguish here between $\bar{z}$ and $z$.

We next introduce the various operators and norms associated with our discretization. With the standard difference operators

$$
\begin{gathered}
D_{+} x(t):=(x(t+h)-x(t)) / h, \quad D_{-} x(t):=(x(t)-x(t-h)) / h, \\
D_{0} x(t):=(x(t+h)-x(t-h)) / 2 h
\end{gathered}
$$


and with

$$
x^{h}: \mathbf{G}^{h} \rightarrow \mathbf{R}, \quad z^{h}: \mathbf{G}_{0}^{h} \rightarrow \mathbf{R}, \quad T^{h}:\left\{\mathbf{G}_{0}^{h} \rightarrow \mathbf{R}\right\} \times \mathbf{R}^{2 n} \rightarrow\left\{\mathbf{G}^{h} \rightarrow \mathbf{R}\right\},
$$

let

$$
\begin{aligned}
\Delta^{h} x & :=x \mid \mathbf{G}^{h}, \quad \hat{\Delta}^{h}(z, r):=\left(z \mid \mathbf{G}_{0}^{h}, r\right), \\
\left\|x^{h}\right\|_{i} & :=\sum_{j=0}^{i} \max \left\{\left|D_{+}^{j} x^{h}\left(t_{\nu}\right)\right| \cdot t_{\nu}, t_{\nu+j} \in \mathbf{G}^{h}\right\}, \\
\left\|z^{h}\right\|_{i} & :=\sum_{j=0}^{i} \max \left\{\left|D_{+}^{j} z^{h}\left(t_{\nu}\right)\right|: t_{\nu}, t_{\nu+j} \in \mathbf{G}_{0}^{h}\right\}, \\
\left\|\left(z^{h}, r\right)\right\|_{i} & :=\left\|z^{h}\right\|_{i}+\|r\|_{0},\|(z, r)\|_{C^{i}}:=\|z\|_{C^{i}}+\|r\|_{0}, \\
\left\|T^{h}\right\|_{0, i} & :=\sup \left\{\left\|T^{h}\left(z^{h}, r\right)\right\|_{i}:\left\|\left(z^{h}, r\right)\right\|_{0} \leq 1\right\} .
\end{aligned}
$$

Clearly $\left\|\Delta^{h} x\right\|_{i} \leq C\|x\|_{C^{i}}$. Choosing difference approximations $\mathscr{D}_{d}^{\nu}$ for $D^{\nu}$ in the differential operators, $\mathscr{D}_{l}^{\nu} x^{h}(0)$ and $\mathscr{D}_{r}^{\nu} x^{h}(1)$ for $D^{\nu} x(0)$ and $D^{\nu} x(1)$ in the boundary operators, we discretize (3.1) into

$$
F_{\alpha}^{h} x^{h}:=\left\{\begin{array}{l}
M_{\alpha}^{h} x^{h}:=\sum_{\nu=0}^{2 n}\left(a_{\nu}\left(t_{i}\right)+\alpha^{2} \delta_{\nu 0}\right) \mathscr{D}_{d}^{\nu} x^{h}\left(t_{i}\right), \quad i=0,1, \ldots, m \\
B^{h} x^{h}:=\left(B_{i}^{h} x^{h}:=\sum_{\nu=0}^{2 n-1}\left[b_{i \nu}(0) \mathscr{D}_{l}^{\nu} x^{h}(0)+b_{i \nu}(1) \mathscr{D}_{r}^{\nu} x^{h}(1)\right]\right)_{i=1}^{2 n} .
\end{array}\right.
$$

Choosing in this formula all the $\mathscr{D}_{s}^{\nu}, s=d, l, r$, by centered compact formulas,

$$
\mathscr{D}_{s}^{2 \nu}:=\left(D_{+} D_{-}\right)^{\nu}, \quad \mathscr{D}_{s}^{2 \nu+1}:=\left(D_{+} D_{-}\right)^{\nu} D_{0} \quad \text { for } s=d, l, r,
$$

we discretize (3.2) into

$$
F_{\alpha}^{h} x_{\alpha}^{h}=\hat{\Delta}^{h}\left(\begin{array}{c}
L_{0}^{*} y \\
\beta_{y}
\end{array}\right) .
$$

For $y \in C^{n+2}[0,1]$, implying $x_{\alpha} \in C^{2 n+2}[0,1]$, we find by standard arguments that

$$
\left\|\hat{\Delta}^{h} F_{\alpha} x_{\alpha}-F_{\alpha}^{h} \Delta^{h} x_{\alpha}\right\|_{0} \leq h^{2} C\|y\|_{C^{n+2}},
$$

and hence, we have consistency independent of $\alpha$. Here and in the sequel we have to assume $0<h<h_{\alpha}$.

To estimate the stability bounds-yielding existence and uniqueness of $x_{\alpha}^{h}$ with (3.6) - we use a result due to Beyn [1], determining the size of this bound instead of showing only its existence as in Grigorieff [8], Kreiss [11], Esser [7], or Vainikko [25]. Let $A_{\alpha}$ and $A_{\alpha}^{h}$ denote the inverse operators for $F_{\alpha}: C^{2 n}[0,1] \rightarrow C^{0}[0,1] \times \mathbf{R}^{2 n}$ and $F_{\alpha}^{h}:\left\{\mathbf{G}^{h} \rightarrow \mathbf{R}\right\} \rightarrow\left\{\mathbf{G}_{0}^{h} \rightarrow \mathbf{R}\right\} \times \mathbf{R}^{2 n}$, respectively. To determine $A_{\alpha}(z, r)$, and hence, to compute the solution $x$ to $F_{\alpha} x=(z, r)$, we use the techniques of Section 2: Let $p:=H N^{\dagger} r, \hat{x}:=x-p \in \mathscr{D}\left(L^{*} L\right),\left(L^{*} L+\alpha^{2} I\right) \hat{x}=z-L_{0}^{*} L_{0} p-\alpha^{2} p$, so finally

$$
A_{\alpha}(z, r):=x=\left(L^{*} L+\alpha^{2} I\right)^{-1}\left(z-L_{0}^{*} L_{0} p-\alpha^{2} p\right)+p .
$$

The continuity of $H N^{\dagger},(2.13)$, and Remark 2.2 yield the estimate

$$
\left\|A_{\alpha}(z, r)\right\|_{C^{i}} \leq\left\|A_{\alpha}(z, r)\right\|_{C^{2 n}}=\|x\|_{C^{2 n}} \leq \frac{C}{\alpha^{2}}\|(z, r)\|_{C^{0}}=\frac{C}{\alpha^{2}}\left\|F_{\alpha} x\right\|_{C^{0}}
$$


for $i=0,1, \ldots, 2 n-1$, which implies that

$$
\left\|A_{\alpha}\right\|_{C^{0, i}}:=\sup _{\|(z, r)\|_{C^{0}=1}}\left\|A_{\alpha}(z, r)\right\|_{C^{i}} \leq \frac{C}{\alpha^{2}} .
$$

Computing $x^{h}$ from $F_{\alpha}^{h} x^{h}=\left(z^{h}, r\right)$ defines $A_{\alpha}^{h}$ by $A_{\alpha}^{h}\left(z^{h}, r\right):=x^{h}$. Then Theorem 6.2 in [1] states that

$$
\lim _{h \rightarrow 0}\left\|A_{\alpha}^{h}\right\|_{0, i}=\left\|A_{\alpha}\right\|_{C^{0, i}} .
$$

In the standard way we obtain from (3.6) and (3.10)

THEOREM 3.1. In (3.1) and (3.2) assume $a_{\nu} \in C^{2}[0,1], \nu=0,1, \ldots, 2 n$, and $y \in C^{n+2}[0,1]$. Then for $i=0,1, \ldots, 2 n-1$,

$$
\begin{gathered}
\left\|\Delta^{h} x_{\alpha}-x_{\alpha}^{h}\right\|_{i} \leq C\|y\|_{C^{n+2}} h^{2} / \alpha^{2}, \\
\left\|\Delta^{h} x_{0}-x_{\alpha}^{h}\right\|_{i} \leq C\|y\|_{C^{n+2}}\left(\alpha^{2}+h^{2} / \alpha^{2}\right) .
\end{gathered}
$$

Remark 3.2. For $P=0$, the negative powers of $\alpha^{2}$ have to be omitted in Theorems 3.1, 4.1, and 4.3, Remarks 3.3 and 4.4, and Corollary 4.2. We will discuss the question of avoiding the negative powers of $\alpha^{2}$ in the context of asymptotic expansions at the end of Section 4.

Remark 3.3. The "generalized Collatz Mehrstellenverfahren" or "Hermitian methods" [6], [10] are defined for the case $a_{\nu}=0, \nu=2 n-1, \ldots, 2(n-p)+$ $3, p>1$. Letting $F_{\alpha}^{h, C}, x_{\alpha}^{h, C}, \Delta^{h, C}, \hat{\Delta}^{h, C}$ denote the appropriate modifications of $F_{\alpha}^{h}, x_{\alpha}^{h}, \Delta^{h}, \hat{\Delta}^{h}$, respectively, we have to solve the equation

$$
F_{\alpha}^{h, C} x_{\alpha}^{h, C}=\hat{\Delta}^{h, C}\left(\begin{array}{c}
L_{0}^{*} y \\
\beta_{y}
\end{array}\right)
$$

corresponding to (3.5). Then

$$
\left\|\hat{\Delta}^{h, C} F_{\alpha} x_{\alpha}-F_{\alpha}^{h, C} \Delta^{h, C} x_{\alpha}\right\|_{0} \leq C h^{2 p}\|y\|_{C^{n+2 p}}
$$

and for $i=0,1, \ldots, 2 n-1$,

$$
\left\|\Delta^{h, C} x_{0}-x_{\alpha}^{h, C}\right\|_{i} \leq C\|y\|_{C^{n+2 p}}\left(\alpha^{2}+h^{2 p} / \alpha^{2}\right) .
$$

4. Asymptotic Expansions for the Discretization Errors. After deriving consistency, stability, and convergence results, we proceed to develop asymptotic expansions for the discretization errors. To allow asymptotic expansions, we need higher smoothness than in Section 3. Consequently, we assume

$$
y \in C^{n+2 q}[0,1] \quad \text { and } \quad a_{\nu} \in C^{2 q}[0,1]
$$

for $\nu=0,1, \ldots, 2 n$, so $x_{0}, x_{\alpha} \in C^{2(n+q)}[0,1]$. Then for the "local discretization error" $\Lambda^{h}$ defined by

$$
F_{\alpha}^{h} \Delta^{h} x=\hat{\Delta}^{h}\left[\left(F_{\alpha}+\Lambda^{h}\right) x\right]
$$


we find the following expression which is independent of $\alpha$ :

$$
\begin{aligned}
\Lambda^{h} x= & \sum_{\mu=1}^{q-1} h^{2 \mu} \widetilde{F}_{2 \mu} x+O\left(h^{2 q}\right)=\sum_{\mu=1}^{q-1} h^{2 \mu}\left(\begin{array}{c}
F_{2 \mu}^{d} x \\
F_{2 \mu}^{b} x
\end{array}\right)+O\left(h^{2 q}\right) \\
= & \sum_{\mu=1}^{q-1} h^{2 \mu}\left(\begin{array}{l}
\sum_{\nu=1}^{2 n} \alpha_{2 \mu}^{\nu} a_{\nu}(t) D^{\nu+2 \mu} x(t) \\
\sum_{\nu=0}^{2 n-1} \alpha_{2 \mu}^{\nu}\left[b_{i \nu}(0) D^{\nu+2 \mu} x(0)+b_{i \nu}(1) D^{\nu+2 \mu} x(1)\right] \\
\end{array} \quad+O\left(h^{2 q}\right),\right.
\end{aligned}
$$

where the $\alpha_{2 \mu}^{\nu}$ are listed as $\alpha_{\mu, \nu}^{e}$ in [10]. In particular,

$$
\alpha_{2 \mu}^{0}=0, \quad \alpha_{2 \mu}^{1}=\frac{1}{(2 \mu+1) !}, \quad \alpha_{2 \mu}^{2}=\frac{2}{(2 \mu+2) !} .
$$

It is obvious that the $\widetilde{F}_{2 \mu}: C^{2 l}[0,1] \rightarrow C^{2(l-\mu-n)}[0,1] \times \mathbf{R}^{2 n}$ are continuous operators for $\mu=1, \ldots, q-1, l \geq n+\mu$.

To compute an asymptotic expansion for the "global discretization error"

$$
x_{\alpha}^{h}-\Delta^{h} x_{\alpha}=\Delta^{h} \sum_{\nu=1}^{q-1} h^{2 \nu} f_{\alpha, 2 \nu}+O\left(h^{2 q}\right),
$$

we determine the $f_{\alpha, 2 \nu}$ recursively from

$$
\left(F_{\alpha}+\sum_{\mu=1}^{q-1}{ }_{r} h^{2 \mu} \tilde{F}_{2 \mu}\right)\left(x_{\alpha}+\sum_{\nu=1}^{q-1} h^{2 \nu} f_{\alpha, 2 \nu}\right)-\left(L_{0}^{*} y, \beta_{y}\right)=O\left(h^{2 q}\right),
$$

where by definition on the left side only terms which are not $O\left(h^{2 q}\right)$ are to be considered, e.g., the term $h^{2 q-4} \widetilde{F}_{2 q-4}\left(h^{6} f_{\alpha, 6}\right)$ is neglected. We have indicated this by the symbol $\sum_{r}$ in (4.4). Using (3.2), we have to compute the $f_{\alpha, 2 \nu}$ inductively from equations which are obtained by annihilating the $h^{2}, h^{4}, \ldots$ terms in (4.4), and hence, for $\nu=1, \ldots, q-1$,

$$
F_{\alpha} f_{\alpha, 2 \nu}=-\left(\widetilde{F}_{2 \nu} x_{\alpha}+\sum_{\mu=1}^{\nu-1} \widetilde{F}_{2 \mu} f_{\alpha, 2(\nu-\mu)}\right):=-\left(\begin{array}{c}
y_{\alpha, 2 \nu} \\
r_{\alpha, 2 \nu}
\end{array}\right) .
$$

Proceeding as in Section 2, we compute

$$
\begin{gathered}
p_{\alpha, 2 \nu}:=-H N^{\dagger} r_{\alpha, 2 \nu} \\
\hat{y}_{\alpha, 2 \nu}:=-\left(y_{\alpha, 2 \nu}+L_{0}^{*} L_{0} p_{\alpha, 2 \nu}+\alpha^{2} p_{\alpha, 2 \nu}\right):=z_{0,2 \nu}+O\left(\alpha^{2}\right), \\
f_{\alpha, 2 \nu}=-\left(L^{*} L+\alpha^{2} I\right)^{-1} \hat{y}_{\alpha, 2 \nu}+p_{\alpha, 2 \nu} .
\end{gathered}
$$

By inductively using the smoothness properties of the $\widetilde{F}_{2 \mu}, x_{\alpha}, f_{\alpha, 2 \nu}$, starting with (4.1), we end up with $\hat{y}_{\alpha, 2 \nu} \in C^{2(q-\nu)}[0,1]$. This implies that

$$
f_{\alpha, 2 \nu} \in C^{2(n+q-\nu)}[0,1] \text {. }
$$

The inductive use of the expansions for $x_{\alpha},\left(L^{*} L+\alpha^{2} I\right)^{-1}, y_{\alpha, 2 \nu}, f_{\alpha, 2 \nu}$, and a careful bookkeeping of norms, necessary extensions, and constants yields the following 
(cf. Remark 3.2)

THEOREM 4.1. Let (4.1) be satisfied and assume the discretization (3.3)-(3.5) is used. Then the functions $f_{\alpha, 2 \nu}$ in (4.4), (4.5) exist for $\nu=1, \ldots, q-1$. With $f_{\alpha, 0}:=x_{\alpha}$ the following estimates are valid for $i=0,1, \ldots, 2 n-1$ :

$$
\begin{aligned}
\left\|x_{\alpha}^{h}-\Delta^{h}\left(\sum_{\nu=0}^{q-1} h^{2 \nu} f_{\alpha, 2 \nu}\right)\right\|_{i} & \leq C \sum_{\nu=0}^{q-1}\left\|f_{\alpha, 2 \nu}\right\|_{C^{2(n+q-\nu)}} \frac{h^{2 q}}{\alpha^{2}} \\
& \leq C\|y\|_{C^{n+2 q}} \frac{h^{2 q}}{\alpha^{2 q}} .
\end{aligned}
$$

The functions $\alpha^{2 \nu} f_{\alpha, 2 \nu}$ admit the following expansions, converging in the norm $\|\cdot\|_{C^{2(n+q-\nu)}}$ :

$$
\alpha^{2 \nu} f_{\alpha, 2 \nu}=\sum_{\mu=0}^{\infty} \alpha^{2 \mu} f_{2 \mu, 2 \nu}, \quad \nu=0,1, \ldots, q-1,
$$

with the norm estimates for $j=-1,0,1, \ldots$ and $\nu=0,1, \ldots, q-1$ :

$$
\left\|f_{\alpha, 2 \nu}-d_{\alpha, 2 \nu, j}\right\|_{C^{2(n+q-\nu)}} \leq C \frac{\left(\alpha^{2} / \alpha_{0}^{2}\right)^{j+1}|j|^{\nu}}{\alpha^{2 \nu}}\|y\|_{C^{n+2 q}}
$$

for $d_{\alpha, 2 \nu, j}:=\sum_{\mu=0}^{j} \alpha^{2(\mu-\nu)} f_{2 \mu, 2 \nu}$.

For the lengthy and highly technical proof, see Theorem 7.1, Corollary 7.2, and Lemma 7.3 in [5].

COROLlaRY 4.2. Under the conditions of Theorem 4.1 define $d_{\alpha, 2 \nu}:=d_{\alpha, 2 \nu, j_{\nu}}$ by choosing bounded $j_{\nu}:=j$ in (4.8). Then the following estimates are valid for $i=0,1, \ldots, 2 n-1$ :

$$
\left\|x_{\alpha}^{h}-\Delta^{h}\left(\sum_{\nu=0}^{q-1} h^{2 \nu} d_{\alpha, 2 \nu}\right)\right\|_{i} \leq C\left(\frac{h^{2 q}}{\alpha^{2 q}}+\sum_{\nu=0}^{q-1} h^{2 \nu} \alpha^{2\left(j_{\nu}+1-\nu\right)}\right)\|y\|_{C^{n+2 q}} .
$$

Proof. Use the triangle inequality on (4.9), combining it with (4.6) and (4.8). Since the $j_{\nu}$ are bounded, $\nu=0,1, \ldots, q-1$, the $\left|j_{\nu}\right|^{\nu} / \alpha_{0}^{2 j_{\nu}+2}$ in (4.8) are bounded as well, independent of $\alpha$ and $h$.

Examining the right-hand side of (4.9), we see that the best we can do is to choose the $j_{\nu}$ such that

$$
h^{2 \nu} \alpha^{2\left(j_{\nu}+1-\nu\right)} \leq C \frac{h^{2 q}}{\alpha^{2 q}} .
$$

Since we want the right-hand side of (4.8) to tend to zero as $h \rightarrow 0$, we have to impose some $h=o(\alpha)$, e.g.,

$$
h=|\alpha|^{c} \quad \text { for } c>1 .
$$

For this case, (4.10) requires us to choose the $j_{\nu}$ such that

$$
j_{\nu} \geq(q-\nu)(c-1)-1 \quad(=q-\nu-1 \text { for } c=2)
$$

for $\nu=0,1, \ldots, q-1$. 
THEOREM 4.3. Let the conditions of Theorem 4.1 and (4.11), (4.12) be satisfied, and choose the $j_{\nu}$ bounded. Then the following inequalities are valid for $i=0,1, \ldots, 2 n-1$ :

$$
\left\|x_{\alpha}^{h}-\Delta^{h}\left(x_{0}+\sum_{\mu=1}^{j_{0}} \alpha^{2 \mu} e_{2 \mu}+\sum_{\nu=1}^{q-1} h^{2 \nu} \sum_{\mu=0}^{j_{\nu}} \alpha^{2(\mu-\nu)} f_{2 \mu, 2 \nu}\right)\right\|_{i} \leq C\left(\frac{h^{2 q}}{\alpha^{2 q}}\right) .
$$

Remark 4.4. Upon using the generalized Collatz Mehrstellenverfahren, (4.13) has to be replaced by

$$
\begin{gathered}
\left\|x_{\alpha}^{h, C}-\Delta^{h}\left(x_{0}+\sum_{\mu=1}^{j_{0}} \alpha^{2 \mu} e_{2 \mu}+\sum_{\nu=p}^{q-1} h^{2 \nu} \sum_{\mu=0}^{j_{\nu}} \alpha^{2(\mu-\nu+p-1)} f_{2 \mu, 2 \nu}\right)\right\|_{i} \\
\leq C \frac{h^{2(q+p-1)}}{\alpha^{2 q}} .
\end{gathered}
$$

One might ask: Under what conditions on the problem and/or its discretization can the negative powers of $\alpha^{2}$ be avoided? Because of the linearity of all operators involved, $f_{\alpha, 2}$ has no $\alpha^{-2}$ term if and only if

$$
P z_{0}=0 \quad \text { with } z_{0}:=z_{0,2}=-F_{2}^{d} x_{0}+L_{0}^{*} L_{0} H N^{\dagger} F_{2}^{b} x_{0} .
$$

This condition may randomly be satisfied. For $\mathscr{N}(L)=\{0\}$ or $P=0$ it is always satisfied. By changing the discretization method, and thus $F_{2}^{d}$ and $F_{2}^{b}$, or $F_{1}^{d}$ and $F_{1}^{b}$ for a first-order method, we again have $P z_{0}=0$ only by pure chance. For problems of the general form (1.1), we have to expect $\mathscr{N}(L) \neq\{0\}$, and hence, a combination of regularization and difference methods unavoidably yields negative powers of $\alpha^{2}$. If one is willing to compute $\mathscr{N}(L)$ first, then one might use the $L^{\dagger}$ given in Section 2 to directly compute $x_{0}$.

5. Numerical Results. The forms of the asymptotic expansions in Section 4 depend strongly upon whether $\mathscr{N}(L) \neq\{0\}$ or $\mathscr{N}(L)=\{0\}$. Whenever this information is available (or probable), e.g., for too few or too many boundary conditions, one should use it. In general, Richardson extrapolation separately for $\alpha$ and $h$ is not appropriate (see [5]), and we relate $\alpha$ and $h$ to obtain an expansion in only one parameter.

In case $\mathcal{N}(L) \neq\{0\}$, a regularization is unavoidable to compute $x_{0}=L^{\dagger} y$, and negative powers of $\alpha^{2}$ occur throughout as in (4.13). Therefore, we choose

$$
\alpha=\sqrt{\gamma h} \quad \text { when } \mathscr{N}(L) \neq\{0\}
$$

to obtain an expansion in powers of $h$ :

$$
x^{h}:=x^{h} \sqrt{\gamma h}=\Delta^{h}\left\{x_{0}+h e_{1}^{*}+h^{2} e_{2}^{*}+\cdots\right\}
$$

with the $e_{i}^{*}$ independent of $h$ and $\alpha$. This situation is illustrated by Example 5.1.

The complementary case $\mathscr{N}(L)=\{0\}$ is only interesting in our context for $\mathscr{N}\left(L^{*}\right) \neq\{0\}$ or $\mathscr{R}(L)=\mathscr{N}\left(L^{*}\right)^{\perp} \neq L^{2}[0,1]$. We choose

$$
\alpha=\gamma h \quad \text { when } \mathscr{N}(L)=\{0\}
$$

to obtain an expansion in powers of $h^{2}$ :

$$
\bar{x}^{h}:=x_{\gamma h}^{h}=\Delta^{h}\left\{x_{0}+h^{2} \bar{e}_{2}+h^{4} \bar{e}_{4}+\cdots\right\}
$$

with the $\bar{e}_{i}$ independent of $h$ and $\alpha$. Example 5.2 illustrates this case. 


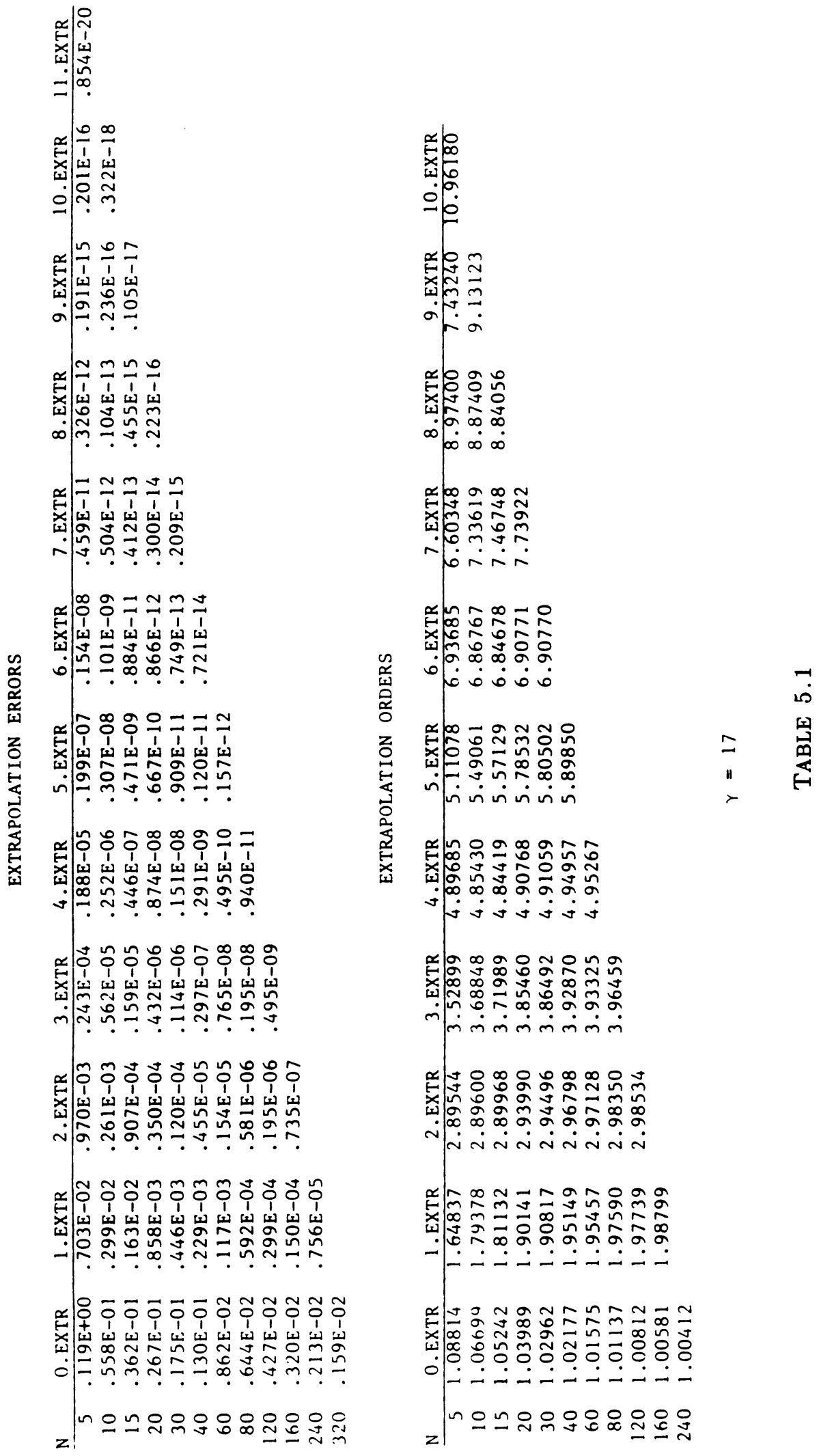




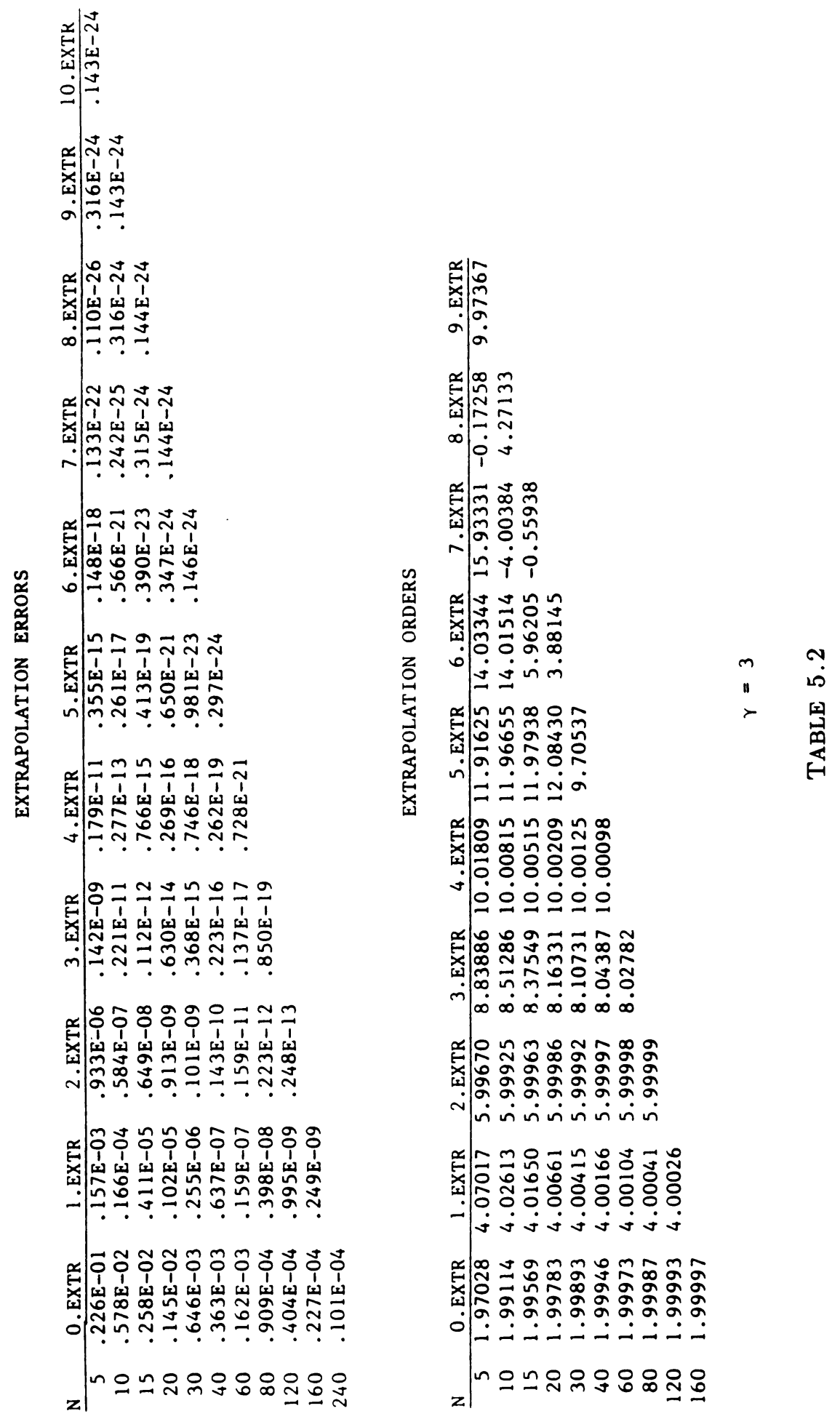


Example 5.1. For $n=k=2$ let

$\mathscr{D}(L):=\left\{x \in H^{2}[0,1] \mid x(0)=x^{\prime}(1)-x(1)=0\right\}, \quad L x:=\left(t^{2}+1\right) x^{\prime \prime}-2 t x^{\prime}+2 x$, and let

$$
y:=6 t^{4}-4 t^{3}+12 t^{2}-12 t+2+t\left(t^{2}+1\right)^{-2},
$$

where $\mathcal{N}(L)=\langle t\rangle, \mathcal{N}\left(L^{*}\right)=\left\langle t\left(t^{2}+1\right)^{-2}\right\rangle$, and

$$
x_{0}=L^{\dagger} y=t^{4}-2 t^{3}+t^{2}-t / 20 \text {. }
$$

Example 5.2. For $n=2, k=3$ let

$$
\mathscr{D}(L):=\left\{x \in H^{2}[0,1] \mid x(1)=x^{\prime}(0)=x^{\prime}(1)=0\right\}, \quad L x:=x^{\prime \prime}+\frac{\pi^{2}}{4} x,
$$

and let $y:=e^{t}$, where $\mathcal{N}(L)=\{0\}, \mathcal{N}\left(L^{*}\right)=\langle\cos \pi t / 2\rangle$, and

$$
x_{0}=L^{\dagger} y=\frac{8}{\pi\left(4+\pi^{2}\right)}\left\{\frac{2}{\pi} \cos \frac{\pi}{2} t-\sin \frac{\pi}{2} t+\frac{\pi}{2} e^{t}-\frac{e \pi-2}{2} t \sin \frac{\pi}{2} t\right\} .
$$

In both examples the right-hand side in $L x=y$ is chosen not to be in $\mathscr{R}(L)$. Utilizing Richardson extrapolation, we have computed the $x^{h}$ and $\bar{x}^{h}$ in (5.1) and (5.2) in quadruple accuracy on the IBM 4381 at the University of Marburg with about 31 digits using the Bulirsch sequence for the stepsizes $h=1 / N$. Tables 5.1 and 5.2 give the results for Examples 5.1 and 5.2, respectively. The error represents the error maximum on the coarsest grid, and the corresponding orders should be $1,2,3, \ldots$ and $2,4,6, \ldots$, respectively.

Acknowledgment. We want to thank cand. math. Zhou Xiaohu, who did the programming, and the referee for his constructive comments.

Fachbereich Mathematik

Universität Marburg

D-3550 Marburg, Federal Republic of Germany

Department of Mathematics

Colorado State University

Fort Collins, Colorado 80523

1. W.-J. BEYN, "Discrete Green's functions and strong stability properties of the finite difference method," Applicable Anal., v. 14, 1982, pp. 73-98.

2. K. BÖHMER, Fehlerasymptotik von Diskretisierungsverfahren und ihre numerische Anwendung, Interner Bericht Nr. 77/2, Institut für Praktische Mathematik, Universität Karlsruhe, 1977.

3. K. BÖHMER, "Discrete Newton methods and iterated defect corrections," Numer. Math., v. 37, 1981, pp. 167-192.

4. K. BÖHMER \& J. LOCKER, "Asymptotic expansions in ill-posed boundary value problems," Z. Angew. Math. Mech., v. 61, 1981, pp. T272-T273.

5. K. BÖHMER \& J. LOCKER, The Mathematical Foundations of Asymptotic Expansions for the Discretization Error of Least Squares Solutions of Linear Boundary Value Problems, Technical Report, Colorado State University, Fort Collins, Colorado, 1984.

6. L. Collatz, The Numerical Treatment of Differential Equations, Springer-Verlag, Berlin and New York, 1966.

7. H. ESSER, "Stabilitätsungleichungen für Diskretisierungen von Randwertaufgaben gewöhnlicher Differentialgleichungen," Numer. Math., v. 28, 1977, pp. 69-100.

8. R. D. GRIGORIEFF, "Zur Theorie linearer approximationsregulärer Operatoren, I und II," Math. Nachr., v. 55, 1973, pp. 233-249 and 251-263. 
9. R. KANNAN \& J. LOCKER, "Continuous dependence of least squares solutions of linear boundary value problems," Proc. Amer. Math. Soc., v. 59, 1976, pp. 107-110.

10. H. B. KelleR \& V. PEREYRA, "Difference methods and deferred corrections for ordinary boundary value problems," SIAM J. Numer. Anal., v. 16, 1979, pp. 241-259.

11. H. O. KREISS, "Difference approximations for boundary and eigenvalue problems for ordinary differential equations," Math. Comp., v. 26, 1972, pp. 605-624.

12. J. LOCKER \& P. M. PRENTER, "Regularization with differential operators. I. General theory," J. Math. Anal. Appl., v. 74, 1980, pp. 504-529.

13. J. LOCKER \& P. M. PRENTER, "Regularization with differential operators. II: Weak least squares finite element solutions to first kind integral equations," SIAM J. Numer. Anal., v. 17, 1980, pp. 247-267.

14. J. LOCKER \& P. M. PRENTER, "Regularization and linear boundary value problems," Applicable Anal., v. 20, 1985, pp. 129-149.

15. F. NAtTereR, "Regularisierung schlecht gestellter Probleme durch Projektionsverfahren," Numer. Math., v. 28, 1977, pp. 329-341.

16. V. PEREYRA, "On improving an approximate solution of a functional equation by deferred corrections," Numer. Math., v. 8, 1966, pp. 376-391.

17. V. PEREYRA, "Iterated deferred corrections for nonlinear operator equations," Numer. Math., v. 10, 1967, pp. 316-323.

18. D. L. PHILLIPS, "A technique for the numerical solution of certain integral equations of the first kind," J. Assoc. Comput. Mach., v. 9, 1962, pp. 84-97.

19. L. F. RichardSON, "The deferred approach to the limit. I: Single lattice," Philos. Trans. Roy. Soc. London Ser. A, v. 226, 1927, pp. 299-349.

20. R. D. RUSSELL, "A comparison of collocation and finite differences for two-point boundary value problems," SIAM J. Numer. Anal., v. 14, 1977, pp. 19-39.

21. R. D. SKEEL, "A theoretical framework for proving accuracy results for deferred corrections," SIAM J. Numer. Anal., v. 19, 1982, pp. 171-196.

22. H. J. STETTER, Analysis of Discretization Methods for Ordinary Differential Equations, Springer-Verlag, Berlin and New York, 1973.

23. A. N. TIKHONOV, "Solution of incorrectly formulated problems and the regularization method," Soviet Math. Dokl., v. 4, 1963, pp. 1035-1038.

24. A. N. TIKHONOV, "Regularization of incorrectly posed problems," Soviet Math. Dokl., v. 4, 1963, pp. 1624-1627.

25. G. VAINIKKO, Funktionalanalysis der Diskretisierungsmethoden, Teubner-Texte zur Mathematik, Teubner, Leipzig, 1976. 